\title{
Original Article \\ Early atherosclerotic lesions of the cardiac conduction system arteries in infants
}

\author{
Luigi Matturri*, Giulia Ottaviani, Anna M. Lavezzi, Lino Rossi \\ Institute of Pathology, University of Milan, Via della Commenda, 19, 20122 Milan, Italy
}

Received 22 October 2003; received in revised form 2 February 2004; accepted 10 May 2004

\begin{abstract}
Introduction: Although several studies have described initial atherosclerotic lesions of the coronary arteries, already detectable in infancy and even during the intrauterine life, little, if any, attention has been given to the possible involvement of the cardiac conduction system arteries. In particular, to the best of our knowledge, none has considered the lesions of the cardiac conduction arteries as an initial stage of atherosclerosis. Methods: The cardiac conduction system of 70 infants dying suddenly and unexpectedly was removed in two blocks for paraffin embedding and serially cut. Results: The histological study of the cardiac conduction arteries of the 70 cases examined showed a normal structure in 55 cases (78.57\%). In 15 cases (21.43\%), there was a thickening of the sinoatrial node and/or atrioventricular artery associated with a thickening of varying severity in coronary artery walls. The lesions were marked by thickening and deposits of amorphous material and mainly lipids in the intima, as well as fragmentation of the elastic fiber system. A significant correlation was evident between early atherosclerotic lesions and both formula feeding and parental cigarette smoking $\left(P<.05, \chi^{2}\right.$ test $)$. Conclusions: The combination of both the considered risk factors seems to increase the early atherogenic effect of each noxa because the coronary lesions were more diffused in formula-fed infants whose parents both smoked. (C) 2004 Elsevier Inc. All rights reserved.
\end{abstract}

Keywords: Atrioventricular node artery; Sinoatrial node artery; Early atherosclerotic lesion; Cardiac conduction system; Cigarette smoking; Formula feeding

\section{Introduction}

Death has climbed in through our windows and has entered our fortresses; it has cut off the children from the streets and the young men from the public squares (Jeremiah 9:21)

Although several studies have described initial atherosclerotic lesions of the coronary arteries, already detectable in infancy [1-4] and even during the intrauterine life [5-7], little, if any, attention has been given to the possible involvement of the cardiac conduction system arteries. In particular, to the best of our knowledge, none has considered the lesions of the cardiac conduction arteries as an initial stage of atherosclerosis.

The finding of early atherosclerotic alterations in infant and fetal coronary arteries $[1-4,6,7]$ prompted us to analyze

\footnotetext{
* Corresponding author. Tel.: +3902 5419522; fax: +39 025419540.

E-mail address: luigi.matturri@unimi.it (L. Matturri).
}

whether there might be an early atherosclerotic involvement also of the cardiac conduction system arteries.

The aim of the present study is to examine the morphologic structure of the cardiac conduction system arteries in 70 infants dying suddenly and unexpectedly.

\section{Material and methods}

\subsection{Selection of cases and method for examination of the cardiac conduction system}

Seventy cases of infants (47 males and 23 females) with unknown medical problems dying suddenly and unexpectedly (SIDS) [8] were selected for this study. Their ages range from 20 to 365 days (mean \pm S.E.M. $=91.90 \pm 9.55$ ).

The clinical data collected for each case included the type of feeding, as well as any parental smoking habit (maternal cigarette smoking before and/or during pregnancy, 
postnatal cigarette smoking by one or both parents and the number of cigarettes smoked daily). The cholesterol level, when available, was within normal range in both infants and mothers.

We have divided the cases into three subgroups: (A) from 0 to 6 weeks; (B) from 7 to 20 weeks; and (C) from 21 to 52 weeks (Table 1).

In each case, a complete autopsy was performed according to the autopsy protocol usually followed by the Institute of Pathology, University of Milan, in case of sudden and unexpected infant death [9], available on the web site: http:// users.unimi.it/ pathol/sids/riscontro_diagnostico_e.html.

Cooperating pathological centers received a brief protocol describing the procedures for the removal and preservation of hearts. The well-known procedure of measuring heart size and weight was not omitted, and the values measured were compared with the normal values for infants of that length and age [10]. After the presence of gross cardiac malformations are excluded, the origin of the coronary arteries were carefully inspected. The hearts were regularly examined for pathologic changes in the atria, septa, ventricles, pericardium, endocardium and coronary arteries. Samples of the myocardium were stained with hematoxylin-eosin and trichromic Heidenhain (Azan).

Our cases were examined in blinded fashion, without initial knowledge of the cause of death, age, or other clinicopathological information. Only after the histological assessment of the conduction system had been completed were the pathological findings matched with the corresponding records.

The cardiac conduction system was removed in two blocks for paraffin embedding [9]. Block 1 consisted of a portion of the right atrial wall including the lateral half of the funnel of the superior venal cava, sulcus and crista terminalis. This first block includes the sinoatrial node, its atrial approaches, the crista terminalis (with the upper 2/3 of the posterior internodal tract, the proximal part of the middle and anterior tracts) and the sinoatrial node ganglionated plexus. Block 2 consisted of the lower portion of the atrial septum, the trigonum fibrosus, the anterior contour of the coronary sinus and the upper $2 / 3$ of the ventricular septum. This second block contains the atrioventricular node, His bundle, bifurcation and bundle branches. For the histological examination of the cardiac conduction system, the two blocks were cut serially at intervals of $40 \mu \mathrm{m}$ (levels), as devised by one of the present authors [11]. For each level, two sections of $8 \mu \mathrm{m}$ were saved and stained alternately with hematoxylin-eosin and Azan. All intervening sections were kept and stained as deemed necessary. For each heart, the average number of histologic sections stained and examined is about 200, as described [12].

\subsection{Coronary and cardiac conduction arteries histologic examination}

According to the protocol followed by Roberts and Jones [13], the hearts were fixed in formalin for at least 1 day. The major epicardial coronary arteries (left main, left anterior descending branch, left circumflex, right posterior interventricular descending branch, right marginal branch) were excised transversely to their longitudinal axis in segments approximately 3-4 mm long. Each segment was labeled sequentially from either its aortic ostium or from its origin from the left main coronary artery. They were dehydrated, embedded in paraffin block and serially cut. The sections of each block were stained with hematoxylin-eosin, Azan for histological examination, Alcian blue (at $\mathrm{pH} 0.5$ and 2.5) for acid mucopolysaccharides analysis, Weigert for elastic fibers identification and, in selected cases, submitted to specific immunohistochemical methods for lymphocyte, monocyte and smooth muscle cell typization. The portions of the sinoatrial and atrioventricular nodal arteries were assessed in the prenodal and intraparenchimal branches, following the serial sections of the two cardiac conduction tissue blocks, without restriction by arterial diameter.

\subsection{Cellular typization of the coronary and cardiac conduction system arteries}

In selected cases, immunophenotyping of the cells present in each preatherosclerotic lesion was performed using the immunoperoxidase technique with the following primary antibodies (made from Dako reagents): anti- $\alpha$ smooth muscle actin to identify smooth muscle cells (SMC),

Table 1

Clinical data and coronary and conduction nodal arteries lesions in infants who died suddenly, divided by postnatal age intervals

\begin{tabular}{|c|c|c|c|c|}
\hline & Subgroup A & Subgroup B & Subgroup C & Total \\
\hline Number of cases & 18 & 39 & 13 & 70 \\
\hline $\operatorname{Sex}(M / F)$ & $13 / 5$ & $23 / 16$ & $11 / 2$ & $47 / 23$ \\
\hline Mean age (days) \pm S.E.M. & $39.13 \pm 3.22$ & $86.16 \pm 3.94$ & $213.4 \pm 25.57$ & $91.90 \pm 9.55$ \\
\hline Parents'smoke & 9 & 19 & 8 & $36(51.42 \%)$ \\
\hline Bottle feeding & 5 & 15 & 8 & $28(40 \%)$ \\
\hline Mixed feeding & 6 & 8 & 3 & $17(24.29 \%)$ \\
\hline Breast feeding & 7 & 16 & 2 & $25(31.71 \%)$ \\
\hline Coronary artery preatherosclerotic lesions & 2 & 16 & 5 & $23(32.85 \%)$ \\
\hline Coronary artery juvenile soft atherosclerotic lesions & - & 6 & 5 & $11(15.71 \%)$ \\
\hline Conduction nodal arteries lesions & 1 & 9 & 5 & $15(21.43 \%)$ \\
\hline
\end{tabular}

Subgroups: A: 0-6 weeks; B: 7-20 weeks; and C: 21-52 weeks. 


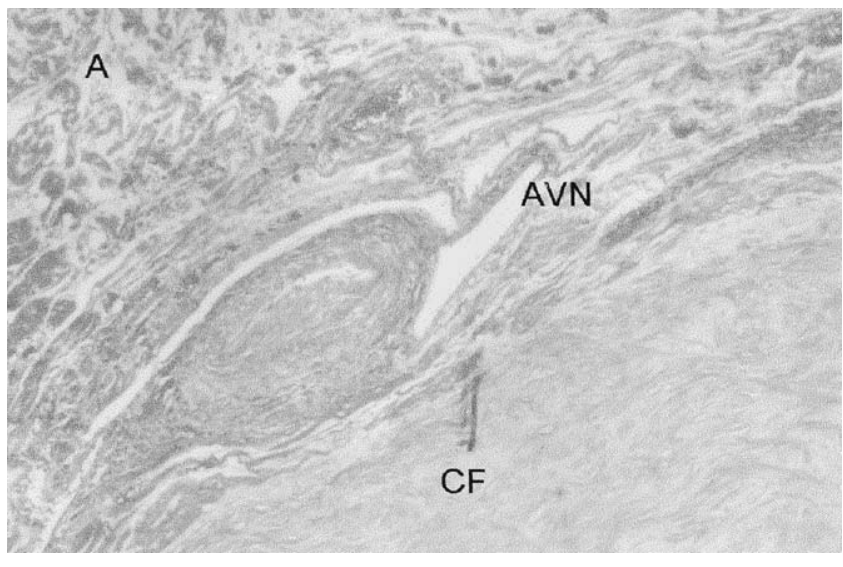

Fig. 1. Narrowing of the atrioventricular node artery in a 6-month-old child dying suddenly and unexpectedly. $\mathrm{A}=$ right atrium; $\mathrm{AVN}=$ atrioventricular node; $\mathrm{CF}=$ central fibrous body. Trichromic Heidenhain; magnification $\times 20$.

anti-CD68 to identify monocytes and anti-CD20 and antiUCHL-1 to identify the B- and T-lymphocytes, respectively.

\subsection{Definition of terms}

(1) Pre-atherosclerotic lesions. The earliest morphologically recognizable alterations are characterized by fragmentation of the tunica media, with intense infiltration of SMC, often arranged perpendicularly in columns or tangentially to the axis of the tunica itself. These SMC infiltrate the intima, which tends to be thickened, also due to initial mucopolysaccharide deposits, mainly composed of Types $\mathrm{A}$ and $\mathrm{C}$ chondroitin sulfates and of hyaluronic acid. Rare monocytes and B-lymphocytes are also present. The endothelium is morphologically intact. The internal elastic membrane appears to be focally fragmented [5].

(2) Juvenile soft atherosclerotic plaques. Such lesions are characterized by wide myointimal thickness with alteration of the elastic fibers and of the tunica media. They are defined as "soft plaques", as they have abundant SMC, associated with the moderate infiltration of monocytes and rare lymphocytes. Amorphous deposits of acid mucopolysaccharides, mainly composed of B chondroitin sulfates, are present, occasionally also in the internal portion of the tunica media. There is evident fragmentation of the elastic fiber system and of the tunica media. The endothelium is morphologically intact. Reduction of the lumen varies from approximately $10-15 \%$ to $30-40 \%$ [14-16].

\subsection{Statistical analysis}

The significance of differences between group parameters were evaluated by $\chi^{2}$ test. The level of significance chosen was $P<.05$, two-tailed.

\section{Results}

\subsection{Cardiac conduction arteries findings}

The histological study of the cardiac conduction arteries of the 70 cases examined showed a normal structure in 55 cases $(78.57 \%)$. In $15 / 70$ cases $(21.43 \%)$, there was a thickening of the sinoatrial node and/or atrioventricular artery associated with a thickening of varying severity in coronary artery walls. The lesions were marked by the infiltration in the intima of SMC, monocytes/foam cells, rare B-lymphocytes and deposits of amorphous material, mainly composed of mucopolysaccharides, as well as focal fragmentation of the elastic fiber system (Figs. 1 and 2). The intima was thickened due to the initial mucopolysaccharide deposits, mainly composed of Types A and C chondroitin sulfates and hyaluronic acid. Rare macrophages and B-lymphocytes were also present.

\subsection{Coronary arteries findings}

In $34 / 70$ cases (48.57\%), a thickening of varying severity in the coronary artery walls was detected (Fig. 3). In 23/70 cases, the lesions were preatherosclerotic, mostly located in the anterior descending branch of the left coronary artery.

In 11/70 cases, juvenile soft atherosclerotic lesions were observed in one or more coronary branches. Such lesions were characterized by deposits of amorphous material, mainly lipids, even in the innermost portion of the media, and significant fragmentation of the elastic fiber system (Fig. 3).

In 19/34 cases, the thickening of the coronary artery was not associated to lesions of the sinoatrial and atrioventricular nodal arteries.

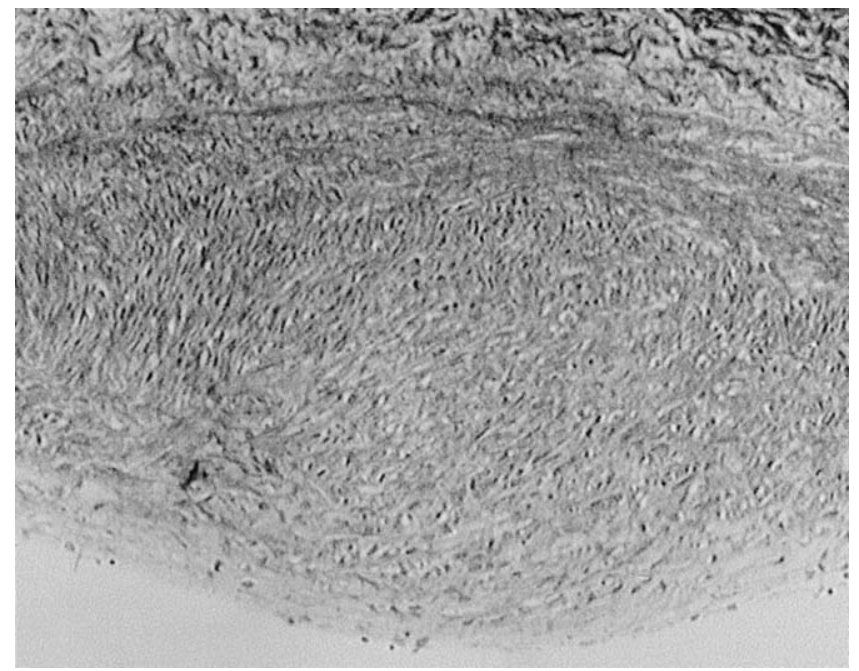

Fig. 2. Fragmentation of the internal elastica lamina in early atherosclerotic lesion. Weigert stain; magnification $\times 200$. 

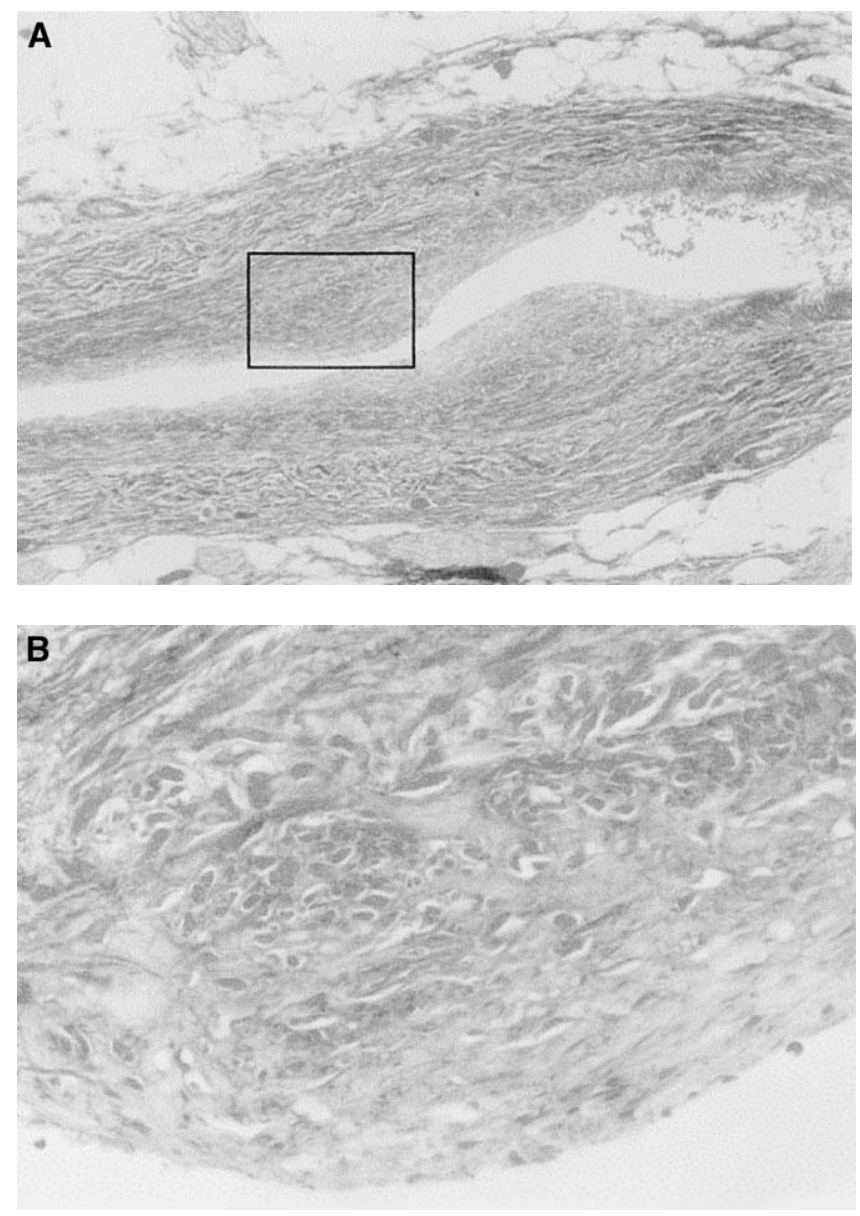

Fig. 3. Histological section of the left circumflex branch of a 10-month-old infant dying of SIDS. Two opposed early atherosclerotic plaques are visible, markedly narrowing the coronary lumen, presenting soft thickening with numerous foam cells. Trichromic Heidenhain; magnification: (A) $\times 25$ and (B) $\times 200$.

\subsection{Feeding type and parents' cigarette smoking}

In 28 cases $(40 \%)$, the infants were fed from birth, or after a few days of breast feeding, only with formula. In 17 cases $(24.29 \%$ ), feeding was mixed (about $50 \%$ formula and $50 \%$ human milk) from birth. Twenty-five babies (31.71\%) were breast fed.

In 36 cases (51.42\%), at least one parent was a smoker and generally smoked over five cigarettes a day. In the smoker mothers, the smoking habit had started before pregnancy.

In 20 cases $(28.57 \%)$, formula feeding was combined with cigarette smoking of one or both parents. The lesions in the cardiac conduction arteries appeared to be larger and with higher number of slides, showing changes when the formula-fed babies had smoker parents ( $9 / 15$ cases, i.e., $60 \%$ of cases).

The clinical data collected disclosed that in 13/15 (86.66\%) infants with intimal thickenings of the cardiac conduction arteries, the feeding was only with formula ( 8 cases) or mixed (5 cases). In 12/15 (80\%) infants with intimal thickening, at least one parent was a smoker.
Among the infants with normal cardiac conduction arteries, $32 / 55(58.18 \%)$ were formula (20 cases) or mixed (12 cases) fed, and in 24/55 (43.63\%), one or both parents were smokers.

A significant correlation is evident between early atherosclerotic lesions and both formula feeding and parental cigarette smoking $\left(P<.05, \chi^{2}\right.$ test).

Regarding age, the infants' cases showed a peak between the 5 th and the 12 th weeks of life. In particular, $21 \%$ of cases occurred at the second month, $22 \%$ at the third, $13 \%$ at the fourth and $10 \%$ at the fifth month. Table 1 shows the clinical data and coronary and conduction nodal artery lesions in infants who suddenly died, divided by postnatal age intervals.

\section{Discussion}

It has been reported that early atherosclerotic or preatherosclerotic lesions are already detectable in infancy [1-4] and even during fetal life [5-7]. Such initial changes are characterized by the thickening of the intima, disruption of the internal elastic lamina, proliferation of the medial SMCs and accumulation of glycosaminoglycans in the fundamental substance of the initial connective tissue $[1,5,13-20]$.

In this study, the histological examination of the serially sectioned cardiac conduction arteries from 70 infants who died suddenly and unexpectedly revealed the frequent presence of early atherosclerotic lesions in 15 of our 70 cases $(21.43 \%)$. The lesions are marked by infiltration in the intima of SMC, monocytes/foam cells, rare B-lymphocytes and deposits of amorphous material, mainly composed of mucopolysaccharides, as well as fragmentation of the elastic fiber system (Fig. 1).

In a recent study [5] carried out on the coronary arteries of human fetuses, we observed early preatherosclerotic alterations in over $50 \%$ of the cases, prevalently on late fetuses of smoker mothers. We interpreted these fetal coronary lesions to have been enhanced by the gaseous products of nicotine.

Fibromuscular hyperplasia or dysplasia [21] of the sinus node and/or AV node arteries has been described as a cause of death in young people and adults [22,23]. Anderson and Hill [24], analyzing 40 victims of crib death, found five cases $(12.5 \%)$ with increased thickness of the AV artery and a case with stenosing thickness of the sinus node artery. The authors hypothesized that this thickness may explain an ischemia of the conducting tissue, with consequent cardiac arrhythmias and/or heart block. Suàrez-Mier and Aguilera [25] reported a fibromuscular hyperplasia in $1.8 \%$ of SIDS cases.

The formula feeding and the parents' cigarette smoking might have a role in the determinism of the herein-described cardiac conduction arteries lesions.

The consequences of baby formula reported in the literature include metabolic disturbances [26-28], delayed 
development of the nervous system [29,30] and dyslipidemia $[31,32]$. In particular, experimental studies on baboons $[33,34]$ and a study on the composition of various types of commercial milk [35] have shown that unlike the fatty acids present in maternal milk, those contained in baby formula cause an increase in the serological cholesterol level, which favors the development of atherosclerosis.

Cigarette smoking has an important atherogenic role and is a major cause of cardiovascular diseases correlated with atherosclerosis in adults [36-39]. Tobacco smoking has been implicated in the initiation and progression of atherosclerotic vascular lesions [31], and recently, we have reported the presence of preatherosclerotic coronary artery lesions in human fetuses of smoker mothers [5]. The combination of both the considered risk factors seems to increase the early atherogenic effect of each noxa because the coronary lesions were more diffused in formula-fed infants whose parents both smoked.

In this study, the relationship between preatherosclerotic lesions and both formula feeding and parental cigarette smoking was statistically significant $\left(P<.05, \chi^{2}\right.$ test $)$.

According to the present findings, we believe that if the results of our series are confirmed by other further studies of the cardiac conduction system on serial sections, the significance of the lesions of the cardiac conduction system arteries would be better understood. Much meticulous clinical-pathologic correlative work between the early atherosclerotic lesions and the risk factors in larger series remains to be done.

\section{Acknowledgments}

This work was supported by the Lombardy Region project 49210-24.3.00: "Program of Research and Intervention for the Reduction of the Risk of Sudden Infant Death and Unexpected Fetal Death". The authors thank Mrs. Graziella Alfonsi, Mrs. Delfina Tosi and Mrs. Lorella Terni for their skillful technical assistance.

\section{References}

[1] Giordano A, Matturri L. Le role des mucopolysaccharides dans l'athérosclérose. Acta Cardiol 1966;11:109-29.

[2] Stary HC. Evolution and progression of atherosclerotic lesions in coronary arteries of children and young adults. Arteriosclerosis 1989;9:119-32.

[3] Peasonen E. Preliminary and early stages of atherosclerosis in childhood. Zentralbl Allg Pathol 1989;135:545-8.

[4] Meyer WW, Lind J, Yao AC, Kauffman SL. Early arterial lesions in infancy and childhood and ways of prevention. Pediatrician 1982:11:136-56.

[5] Matturri L, Lavezzi AM, Ottaviani G, Rossi L. Intimal preatherosclerotic thickening of the coronary arteries in human fetuses of smoker mothers. J Thromb Haemost 2003;1:2234-8.

[6] Neufeld HN, Wagenfoort CA, Edwards JE. Coronary arteries in fetuses, infants, juveniles and young adults. Lab Invest 1962;11:837-44.
[7] Lehtovirta P, Pesonen E, Sarna A. Effect of smoking on the fetal coronary arteries. Acta Pathol Macrobiol Immunol Scand A 1984; 92:189-93

[8] Willinger M, James S, Catz C. Defining the SIDS: deliberations of an expert panel convened by the National Institute of Child Health and Human Development. Pediatr Pathol 1991;11:127-32.

[9] Matturri L, Ottaviani G, Ramos SG, Rossi L. Sudden infant death syndrome (SIDS): a study of cardiac conduction system. Cardiovasc Pathol 2000;9:137-45.

[10] Gilbert-Barness E. Potter's pathology of the fetus and infant St. Louis: Mosby Year Book, 1997.

[11] Rossi L. "Salvage the pacemaker" at autopsy. Am Heart J 1978;95:540-1.

[12] Ottaviani G, Matturri L, Rossi L, James TN. Crib death: further support for the concept of fatal cardiac electrical instability as the final common pathway. Int J Cardiol 2003;92:17-26.

[13] Roberts WC, Jones AA. Quantitation of coronary arterial narrowing at necropsy in sudden coronary death: analysis of 31 patients and comparison with 25 control subjects. Am J Cardiol 1979;44: $39-45$.

[14] Matturri L, Ottaviani G, Corti G, Lavezzi AM. Pathogenesis of early atherosclerotic lesions in infants. Pathol Res Pract 2004, in press.

[15] Lavezzi AM, Milei J, Grana DR, Flenda F, Basellini A, Matturri L. Expression of c-fos, p53 and PCNA in the unstable atherosclerotic plaque. Int J Cardiol 2003;92:73-7.

[16] Stary HC. Evolution and progression of atherosclerotic lesions in coronary arteries of children and young adults. Arteriosclerosis 1989; 9:119-32.

[17] Pesonen E. Preliminary and early stages of atherosclerosis in childhood. Zentralbl Allg Pathol 1989;135:545-8.

[18] Meyer WW, Lind J, Yao AC, Kauffman SL. Early arterial lesions in infancy and childhood and ways of prevention. Pediatrician 1982; 11:136-56.

[19] Schornagel HE. Intimal thickening in the coronary arteries in infants. Arch Pathol 1956;62:427-32.

[20] Robertson JH. The significance of intimal thickening in the arteries of the newborn. Arch Dis Child 1960;35:588-90.

[21] James TN. Congenital disorders of cardiac rhythm and conduction. J Cardiovasc Electrophysiol 1993;4:702-18.

[22] James TN, Marshall TK. De subitaneis mortibus XVII: Multifocal stenoses due to fibromuscolar dysplasia of the sinus node artery. Circulation 1976;53:736-42.

[23] James TN, Froggat P, Marshall TK. Sudden death in young athletes. Ann Intern Med 1967;67:1013-21.

[24] Anderson KR, Hill RW. Occlusive lesions of cardiac conducting tissue arteries in sudden infant death syndrome. Pediatrics 1982;69: $50-2$.

[25] Suàrez-Mier MP, Aguilera B. Histopathology of the conduction system in sudden infant death. Forensic Sci Int 1998;93:143-54

[26] Fifty-fifth World Assembly Health, 13-18 May 2002 (http// www.who.int $/ \mathrm{gb} /$ ).

[27] De Rooy L, Hawdon J. Nutritional factors that affect the postnatal metabolic adaptation of full-term small- and large-for-gestational-age infants. Pediatrics 2002;109:E42.

[28] McGill HC, Mott GE, Lewis DS, McMahan CA, Jackson EM. Early determinants of adult metabolic regulation: effects of infant nutrition on adult lipid and metabolism lipoprotein. Nutr Rev 1996; $54: 531-40$

[29] Amin SB, Merle KS, Orlando MS, Dalzell LE, Guillet R. Brainstem maturation in premature infants as a function of enteral feeding type. Pediatrics 2000;106:318-22.

[30] Jamieson EC, Farquharson J, Logan RW, Howatson AG, Patrick WJ, Weaver LT, Cockburn F. Infant cerebellar gray and white matter fatty acids in relation to age and diet. Lipids 1999;34:1065-71.

[31] Wagner V, von Stockhausen HB. The effect of feeding human milk and adapted milk formulae on serum lipid and lipoprotein levels in young infants. Eur J Pediatr 1988;147:292-5. 
[32] Tammi A, Ronnemaa T, Viikari J, Jokinen E, Lapinleimu H, Ehnholm C, Simell O. Apolipoprotein E4 phenotype increases nonfasting serum triglyceride concentration in infants-the STRIP study. Atherosclerosis 2000;152:135-41.

[33] Mott GE, McMahan CA, Kelley JL, Farley CM, McGill HC. Influence of infant and juvenile diet on cholesterol serum, lipoprotein cholesterol and apolipoprotein concentrations in juvenile baboons. Atherosclerosis 1982;45:191-202.

[34] Mott GE, Lewis DS, McGill HC. Programming of cholesterol metabolism by breast of feeding formula. Ciba Found Symp 1991;156:56-76.
[35] Droese W, Pape E, Stolley H. Questions to the supply of young infants with fat and fatty acids. Eur J Pediatr 1976;123:277-92.

[36] McGill HC. The cardiovascular pathology of smoking. Am Heart J 1988;115:250-7.

[37] Lakier JB. Smoking and cardiovascular disease. Am J Med 1992;93:88-128.

[38] Weintraub WS. Cigarette smoking as a risk factor for coronary artery disease. Adv Exp Med Biol 1990;273:27-37.

[39] Burns DM. Epidemiology of smoking-induced cardiovascular disease. Prog Cardiovasc Dis 2003;46:11-29. 